\title{
On the Complexity of "Always Best Connected" in 4G Mobile Networks
}

\author{
Vangelis Gazis, Nikos Houssos, Nancy Alonistioti, Lazaros Merakos \\ Communication Networks Laboratory, Department of Informatics \& Telecommunications, \\ University of Athens, 157 84, Athens, Greece, \\ email: \{gazis, nhoussos, nancy, merakos\}@di.uoa.gr
}

\begin{abstract}
Global developments in wireless communications have shaped a novel, user-centric vision for the next generation of mobile systems and wireless access network broadly termed "4G". This widely accepted vision sketches a heterogeneous infrastructure comprising different wireless access systems in a complementary manner, where the user, supported by his/her personal intelligent agent(s), enjoys untethered connectivity and ubiquitous access to applications, value-added services and multimedia content over the most efficient combination of wireless and wireline systems available. We proceed to analyze the implications of this "Always Best Connected" vision to identify pivotal concepts and formulate an appropriate model. Furthermore, we proceed to analyze the complexity of our proposed model by showing that, in principle, being "Always Best Connected" translates to family of problems that are known to be $N P$-hard.
\end{abstract}

Keywords-Mobile; wireless; 4G; service selection; utility

\section{INTRODUCTION}

Fourth generation mobile communications (i.e., 4G) tend to mean different things to different people: for some it is merely a higher-capacity (e.g., $100 \mathrm{Mb} / \mathrm{s}$ ) new radio interface, while for others it is an interworking of cellular and wireless LAN (WLAN) technologies that employs a variant of the Mobile IPv6 mobility management protocol (e.g., Hierarchical Mobile IPv6) for inter-system handoff and IETF AAA technologies for seamless roaming. There is no doubt that fourth generation mobile communication systems will provide even higher data rate services or that IETF-bred technologies will constitute fundamental building blocks of future wireless infrastructures. Traffic demand estimates [1] [2] suggest that, to accommodate the foreseen amount of traffic in the $2010-2020$ timeframe in an economically viable way, $4 \mathrm{G}$ mobile systems must achieve a manifold increase in capacity compared to their predecessors.

In the European Union, the debate about $4 \mathrm{G}$ mobile systems has been taking place mostly within the context of its IST Framework Program activities. Out of this process has spawned the vision of a system that enables an "Always Best Connected" - or "ABC" for short - mode of communication for the citizen of the forthcoming information society [3]. This now widely accepted vision sketches a heterogeneous communication infrastructure comprising different wireless access systems (e.g., GSM/GPRS, UMTS, DVB-T, HAPS, WLAN) in a complementary manner, where the user, supported by his/her personal intelligent agent(s), enjoys untethered connectivity and ubiquitous access to applications over the most efficient combination of available systems. Thus, $4 \mathrm{G}$ primarily marks a paradigm shift from a technology-driven towards a user-focused systematic approach.

Asian and North American viewpoints on the scope, shape and structure of $4 \mathrm{G}$ mobile systems are aligned to the European Union one and vision streamlining is jointly pursued by the ITU-T Special Study Group on "IMT-2000 and Beyond" [1]. Fig. 1 illustrates the future 4G mobile network architecture comprising ad-hoc, hot-spot, cellular, high-altitude and satellite radio components.

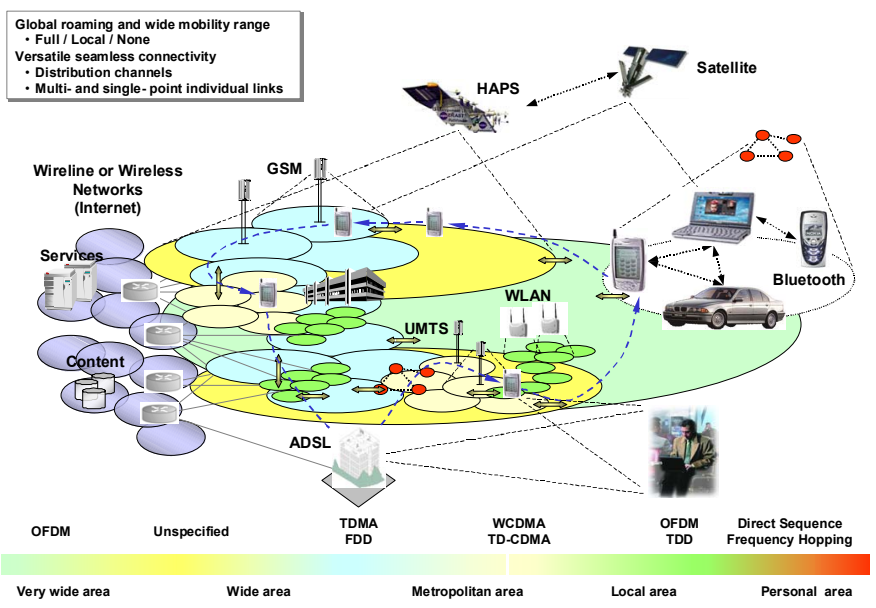

Figure 1. The heterogeneous multi-standard 4G mobile network architecture.

4G will be characterized by a horizontal communication model, where different access technologies such as cellular, cordless, wireless LAN type systems, short range connectivity and wired systems will be combined on a common platform to complement each other in an optimum way for different service requirements and radio environments. These different (wireless and/or wireline) access networks will interface to core and/or backbone network elements over the IP protocol, the lingua franca of networking technology. Thus, the prime objective for $4 \mathrm{G}$ mobile systems is to incorporate and integrate different wireless access technologies and mobile network architectures in a complementary manner so as to achieve a seamless wireless access infrastructure.

The rest of the paper is structured as follows: Section II identifies key concepts of $4 \mathrm{G}$ mobile communications and 
highlights important implications of the heralded "Always Best Connected" concept. Section III introduces our "ABC" model, and analyzes its complexity, by showing that, in principle, being "Always Best Connected" belongs to a popular class of $N P$-hard combinatorial optimization problems. Finally, section IV concludes the paper with directions for future work.

\section{IMPORTANT CONCEPTS IN 4G MOBILE ENVIRONMENTS}

\section{A. User-centric view of "Always Best Connected"}

The interworking of different radio technologies and access network architectures in a multi-coverage environment results in a proliferation of service delivery options for IP-based mobile applications (e.g., location-based services and VoIP applications). Which of these options will be employed by the system depends on numerous configuration parameters - that are possibly under different administrations (e.g., GSM/UMTS network operator, WLAN network operator) - that collectively affect the behavior of multiple network elements (e.g., mobile terminal, base station, etc) in different administrative domains. The widely accepted user-centered "ABC" vision for $4 \mathrm{G}$ requires that all such important parameters and configuration options be provisioned in a coordinated manner according to each user's preferences. For instance, in a UMTS and WLAN radio coverage scenario, transporting a media stream to the mobile device can be accomplished by either of these wireless systems. However, the decision regarding which particular system(s) to use will depend on a number of factors, such as the respective cost of service, availability of network resources, radio link quality, service requirements and user preferences. One could imagine scenarios where the end-to-end signaling between application endpoints is routed via UMTS, because of its predictable performance and quality of service guarantees, while the media stream is routed via a nearby wireless LAN to take advantage of its significantly greater bandwidth capacity.

\section{B. Multi-access and context-awareness}

Such "access network selection" decisions will depend on the communication requirements of end-user applications their relative importance to the mobile user and the tasks he/she is currently engaged in. In other words, such decisions should be context-dependent [4] and any decision-making algorithms will have to be context-aware as well as user-aware. Furthermore, system decision support must be applicable to a wide range of user applications, from the ubiquitous web browser and VoIP applications to advanced location-based services and enterprise applications. continuing our previous example with a focus on the cost factor, the monetary cost of downloading a file over an FTP connection will depend on the pricing model and charging formula applied by the wireless access network (i.e., WLAN or UMTS) employed to transport the FTP traffic. Given the large variety of possible charging formulae, break-even analysis could be used to select the cheapest wireless access network on a per-file basis for such applications (i.e., whose performance affected by a single QoS dimension). Table I illustrates a hypothetical pricing configuration of four different wireless access networks, a UMTS and a WLAN ${ }^{1}$, run by four different

\footnotetext{
${ }^{1}$ Example WLAN technologies that support QoS management for access bearers are HiperLAN/2 and IEEE 802.11e
}

network operators that employ volume-based charging for user IP traffic. We note that support for such volume-based pricing models is standard in UMTS [5] and may be readily applied in WLAN technologies (e.g., the UTMS-WLAN interworking architecture for UMTS R6).

TABLE I. EXAMPLE PRICING MODELS OF WIRELESS ACCESS

\begin{tabular}{|c|c|c|c|c|}
\hline \multirow{2}{*}{$\begin{array}{c}\text { Wireless } \\
\text { Access }\end{array}$} & \multicolumn{3}{|c|}{ Cost configuration } \\
\cline { 3 - 5 } & Pricing formula & Data volume & Cost \\
\hline 1 & UMTS & $1.0 €+1.0 € / \mathrm{MB}$ at $64 \mathrm{~KB} / \mathrm{s}$ & $10 \mathrm{MB}$ & $11.0 €$ \\
\hline 2 & UMTS & $0.7 €+2.5 € / \mathrm{MB}$ at $128 \mathrm{~KB} / \mathrm{s}$ & $10 \mathrm{MB}$ & $25.7 €$ \\
\hline 3 & WLAN & $2.0 €+0.5 € / \mathrm{MB}$ at $256 \mathrm{~KB} / \mathrm{s}$ & $10 \mathrm{MB}$ & $12.0 €$ \\
\hline 4 & WLAN & $3.0 €+1.0 € / \mathrm{MB}$ at $512 \mathrm{~KB} / \mathrm{s}$ & $10 \mathrm{MB}$ & $18.0 €$ \\
\hline
\end{tabular}

Assuming for the moment that "Always Best Connected" translates merely to identifying and using the cheapest access network, Table I illustrates that selecting just one access network does not always yield an optimum solution. In this case, the optimum (i.e., the cheapest) solution results by employing both access networks; a UMTS $64 \mathrm{~Kb} / \mathrm{s}$ service for the first $3 \mathrm{MB}$ of data and a WLAN $256 \mathrm{~Kb} / \mathrm{s}$ service for the remaining $7 \mathrm{MB}$ of data. Although this example may seem farfetched, it demonstrates the (inherently) context-dependent nature of the "Always Best Connected" concept in multi-access environments.

\section{Personalization}

In the case of multi-dimension QoS applications and multiple different pricing models (e.g. volume-based, flat-rate location-based, duration-based, etc) various contextual settings and parameters may have to be resolved and employed in a decision-making algorithm that will provide an optimal - if not optimum - solution to the instantaneous "ABC" problem of each particular user. Considering the imperative need for personalization in mobile service provision [6], user preferences will constitute an integral part of such contextual information. Consider, for example, the case of a wireless access network capable of providing network-based VPN services to roaming users [7]. Business users wishing to access corporate databases with their mobile device (e.g., laptop computer) may be willing to pay a premium price for the greater assurance levels the VPN service offers, while typical users browsing for content may be comfortable will a lower security level - and cost. User preferences however, are hard to capture and express, mainly because they cannot be computed; only revealed by informed user choices. User preferences are among the foundations of microeconomic theory, which uses the utility concept as a continuous function representation of the consumer's preference relation over a set of commodities [8].

\section{User and application utilities}

Users consume services and employ applications to realize various benefits, and as long as these (subjective) benefits are perceived to overbalance the respective charges, users will continue consumption and use. Communication-based services 
and applications depend on the timely and orderly provision of network bearer services to exchange application-specific signaling between remote application entities and to transport various forms of user information (e.g., image, video, corporate data, etc) between communicating application endpoints. The performance of such communication-based applications is dependent on the accommodation of quality of service requirements for their native signaling as well as for the transport of arbitrary user information. From a network viewpoint, these factors translate to traffic flows with different quality of service requirements that will levy different charges, depending on which particular access network(s) will be employed to transport them, thus decreasing user satisfaction. Ensuring an adequate performance level for communicationbased applications so as to maximize user satisfaction, requires honoring the quality of service requirements of their traffic flows while minimizing the overall charges incurred, i.e., solving the user's utility maximization problem. Notably, bearer service providers face the dual problem, i.e., maximizing revenue and minimizing network resource usage whilst satisfying quality of service requirements for all serviced traffic flows. Users, however, are oblivious and indifferent to the resource management implications of application usage in a mobile device. All they are interesting in are the (subjective) perceived benefits they enjoy from using their applications and the monetary cost of accruing these benefits. Thus, for any given combination of user application, traffic flow and quality of service level, user utility will decrease as the monetary cost of enjoying that combination increases. Simply put, users will consider "worst" any access selection decision that leads to a higher monetary cost. However, as our example in Table I demonstrated, monetary cost may depend on arbitrary traffic flow properties, and thus, the lowest monetary cost may be attainable only through the combined use of multiple wireless access networks.

\section{AN “Always Best CONNECTED” MODEL}

\section{A. Related work}

[9] considers the case of heterogeneous wireless access from the viewpoint of policy decisions regarding the optimal radio access technology to hand off to based on a set of criteria. [10] proposes an agent-based middleware system that mediates between mobile users, wireless network operators and service (i.e., application) providers to negotiate QoS contracts for userconsumed services (and the respective traffic flows) between the mobile user device and the wireless access networks its has roamed to. Negotiation proceeds by a bidding process that satisfies the requirements of accessibility, transparency and accountability necessary to form a free (digital) marketplace. Their concept is very much in line with ours, however [10] does not attempt a complexity analysis of the proposed approach. [11] addresses the subjective and objective nature of the "ABC" concept from the viewpoints of the various stakeholders in the mobile value chain (e.g., mobile user, network operator, etc) and proposes a model for the user's best connectivity solution that incorporates the cost function of [9]. Notably, it also embraces the premise that the user's viewpoint is of prime importance in seeking an efficient solution to the "ABC" problem. It also acknowledges that, contrary to the current inflexible practice, future mobile systems may dynamically select which wireless access network(s) to use on a per session - or even call - basis. To summarize, although most existing efforts recognize the policy implications of access selection decisions in multi-access environments, using some - direct or proxy - measure of user utility, they all assume that the respective cost of access is constant and a priori known. Our formulation of the "ABC" model adopts a user-centric approach that does not make any assumptions on the access cost other than that is calculated by a formula specific to each (wireless or wireline) access network.

\section{B. The proposed "Always Best Connected" model}

Mobile devices must cope with the unpredictable nature of the wireless medium which, may exhibit rapid QoS fluctuation, or even total service unavailability. Given that the greatest challenge in heterogeneous multi-access wireless environments is the wide range or operational parameters [12], adaptability is recognized as an important property of future wireless systems [13]. Adaptation strategies may be applied to functional network elements so as to dynamically adjust their grade of service according to specific application criteria. For instance, in the case of an adaptable multimedia streaming application whose bandwidth requirements cannot be met by the current wireless bearer service, the possible adaptation strategies are a) to lower the video and audio codec resolutions, b) to negotiate an higher bandwidth for the bearer service or c) to do a combination of a) and b). Adaptability facilitates fine-grain control over system and network resources expedited by active application(s) within a mobile device, whose resources (e.g., power supply, processing power) are finite and thereby, precious. Thus, our "ABC" model expands on earlier work in the filed of adaptive QoS management: [14], that proposes a utility model to capture the issues of resource management within an adaptive multimedia system with multiple concurrent sessions where the quality of individual sessions is dynamically adapted to available resources and user preferences, and [15], that tackles the problem of maximizing system utility by allocating a finite resource to meet the QoS requirements of multiple applications along multiple QoS dimensions. Our formulation of the "ABC" problem is based on the following concepts:

- Each user application requires the transport of a set of traffic flows to function.

- Each application traffic flow may be accommodated at different quality of service levels.

- Each QoS level is uniquely mapped to the amount of computing and communication resources required to support that QoS level.

- Computing and communication resources are finite.

- Application utility is the sum of the utilities of all its traffic flows.

- User utility is the sum of the utilities of all its active applications. 
We adopt the common assumption that each application $A_{i}, i=\{1,2, \ldots, N\}$, that is active at the user' mobile terminal will employ a set $F\left(A_{i}\right)=\left\{f_{i 1}, f_{i 2}, \ldots, f_{i F_{i}}\right\}$ of unidirectional traffic flows in communicating with its peer endpoints. We consider the set $F\left(A_{i}\right)$ to be dynamically volatile, i.e., an application may voluntarily increase or decrease the number of its traffic flows as it desires, establishing new sessions or terminating existing ones - within resource limitations of course. We regard QoS a multi-dimensional space defined over a collection of performance metrics (e.g., bandwidth, mean delay, mean error rate, etc) that is common across all applications and interoperable to the quality of service scheme of each individual wireless access network. These QoS dimensions will be ranked differently, by different end-user applications; e.g., a multimedia streaming application will rank end-to-end delay higher than information integrity (i.e., mean error rate), while an enterprise application will probably rank information integrity the highest. Let $Q$ to denote the QoS space and $q$ a point in the QoS space (i.e., a QoS level), while $R$ denotes the resource space and $r$ a point in the resource space (i.e., an amount of resources). Then, $q_{i j}=\left\{q_{1}^{i j}, \ldots, q_{N(i j)}^{i j}\right\}$ is the QoS profile of flow $f_{i j}$, where $N(i j)$ is the number of QoS levels for the flow.

As in [14] [15], we assume the existence of the following mappings:

- Quality-to-resource mapping $R(q): Q \rightarrow R$, which maps from the quality of service level of a traffic flow to the resource levels required to provide that quality.

- Quality-to-utility mapping $U(q): Q \rightarrow[0,1] \subseteq \Re$, which maps from the quality of service level of a traffic flows to the resource levels required to provide that quality.

In addition to [14] [15], we also assume the existence of the following mapping:

- $\quad$ Resource-to-cost mapping $C(r): R \rightarrow C$, which maps from a specific resource level to the monetary cost of committing the resource at that particular level. We use the resource-to-cost mapping to capture the difference in the pricing model employed by each wireless access network and as a determinant of the monetary cost of each particular traffic flow.

We consider that application and user utilities result from the utilities of the individual traffic flows, as follows:

$$
\begin{aligned}
& \text { Application } A_{\mathrm{i}} \text { utility: } U\left(A_{i}\right)=\sum_{j=1}^{F_{i}} u\left(q_{i j}\right) \\
& \text { User } u \text { utility: } U=\sum_{i=1}^{N} U\left(A_{i}\right)=\sum_{i=1}^{N} \sum_{j=1}^{F_{i}} u\left(q_{i j}\right)
\end{aligned}
$$

The marginal user utility $u\left(q_{i j}\right)$ induced by the transport of traffic flow $f_{i j}$ at QoS level $q_{i j}$ will be decreased by the monetary cost $c_{i j}$ of the resources employed to guarantee the desired quality of service $q_{i j}$ for that particular traffic flow. Thus, the marginal utility, positive or negative, will be equal to:

$$
u_{i j}^{m \text { arg inal }}\left(f_{i j}\right)=u\left(f_{i j}\right)-c\left(f_{i j}\right)=u\left(q_{i j}\right)-c\left(q_{i j}\right)
$$

Our formulation considers as "Always Best Connected" any solution to the user's utility maximization problem, where the user utility is $u_{i j}^{m \text { arginal }}\left(f_{i j}\right)=u^{m \text { arginal }}\left(q_{i j}\right)$.

\section{Knapsack problems}

Knapsack problem represent an entire family of problems that require a subset of some given items to be chosen such that the corresponding profit sum is maximized without exceeding the capacity of the knapsack(s) [16]. The typical Knapsack problem is formulated as follows: Consider a set of $N$ objects $O=\left\{o_{1}, \ldots, o_{N}\right\}$ and a knapsack of capacity $C$. Each object $o_{i}$ has weight $w_{i}$ and results in profit $p_{i}$ when wholly included in the knapsack. If a fraction $x_{i}, x_{i} \in[0,1]$ of object $o_{i}$ is placed into the knapsack then a profit of $x_{i} p_{i}$ is accrued and the available knapsack capacity is decreased by $x_{i} w_{i}$. The objective is to obtain a filling of the knapsack that maximizes the total profit earned without overflowing the knapsack. Formally, the problem can be stated as:

$$
\text { Maximize } Z=\sum_{i=1}^{N} p_{i} x_{i} \text { s.t. } \sum_{i=1}^{N} w_{i} x_{i} \leq C
$$

Various classes of the Knapsack problem exist, like the 0-1 Knapsack problem, which occurs when the decision variables $x_{i} \in\{0,1\}$ and the multiple choice multiple dimension (MMKP) Knapsack problem which arises when there are $G$ groups of items with $N_{i}$ items in each group and exactly one item to be selected from each group for inclusion in the knapsack. A usual variation of the $0-1$ and MMKP Knapsack problems is the use of multiple knapsacks $K=\left\{K_{1}, \ldots, K_{M}\right\}^{2}$. The generalized form of the Knapsack problems is the Generalized Assignment Problem (GAP), which is stated as follows: Consider a set of items $O=\left\{o_{1}, \ldots, o_{N}\right\}$, and a set of knapsacks $K=\left\{K_{1}, \ldots, K_{M}\right\}$, where knapsack $K_{j}$ has capacity $c_{j}$. Let $p_{i j}$ be the profit accrued if object $o_{i}$ is wholly placed in knapsack $K_{j}$ and weight $w_{i j}$ the respective decrease to the knapsack capacity. The GAP seeks an assignment of objects to knapsacks such that the total profit is maximized without overflowing any of the knapsack(s):

$$
\begin{aligned}
& \text { Maximize } Z=\sum_{i=1}^{N} \sum_{j=1}^{M} x_{i j} p_{i j}, x_{i j} \in\{0,1\} \\
& \text { s.t. } \sum_{i=1}^{N} \sum_{j=1}^{M} x_{i j} w_{i j} \leq C_{j}, \forall j \in\{1, \ldots, M\}
\end{aligned}
$$

The GAP is known to be NP-complete, while the problem of deciding if there exists a feasible solution (i.e., one that accommodates all items) is $N P$-hard [16].

For an excellent categorization of Knapsack problems see [15] 


\section{Always Best Connected as a Knapsack problem}

Assuming for a moment that the resource-to-cost mappings are the same for all accessible wireless access networks, our "ABC" model can be isomorphically mapped to the MMKP Knapsack problem with multiple knapsacks as follows:

- The number of traffic flows $F=\sum_{i=1}^{N} F_{i}$ in mapped to the number of groups of items.

- The QoS profile $q_{i j}=\left\{q_{1}^{i j}, \ldots, q_{P}^{i j}\right\}$ of each traffic flow $f_{i j}$ is mapped to a group of items, exactly one of which will be placed in a knapsack $K_{j}$.

- Each accessible access network is mapped to a knapsack ${ }^{K}$ of capacity ${ }^{c_{j}}$.

- Total user utility is mapped to the profit accrued by the items that are included in the knapsacks.

- The resource requirements, i.e., the values $r\left(q_{i j}\right) \in R$ that result from the QoS-to-resource mappings for each QoS level $q_{i j}$, are mapped to the weight $w_{i j}$ of the items that are placed in the knapsacks.

- The resource constraints of the "ABC" problem are mapped to the resource constraints (i.e., the capacity) of the knapsacks.

- Choosing the QoS level is viewed as picking exactly one item from each group to include in a knapsack.

Thus, with constant resource-to-cost mappings, our "ABC" model maps to an MMKP Knapsack problem with multiple knapsacks, which is known to be NP-hard [16]. Reverting back to the original formulation where the user utility induced by each traffic flow is dependent not only upon the particular QoS level assigned to the traffic flow, but also upon the respective cost-to-resource mapping applied by the access network chosen to transport the flow, our "Always Best Connected" model is isomorhically mapped to the GAP problem, which is also known to be $N P$-hard. Despite the exponential complexity, approximation algorithms that compute an optimal solution within a given bound distance from the optimum solution, may be employed to reduce computation time [16]. We should note that, because of the duality property, wireless access network operators face problems of equal complexity, e.g., deciding which particular flows to admit in their network with as little a resource expenditure as possible.

\section{CONCLUSIONS AND FUTURE WORK}

In the heralded $4 \mathrm{G}$ mobile environment, numerous wireless access technologies will coexist, complementing each other as well as wireline access solutions. Vision consensus regarding the function of $4 \mathrm{G}$ mobile service provision suggests that $4 \mathrm{G}$ should operate in a transparent user-centric way, as opposed to the technology-centric way of its predecessors. Analyzing and understanding what the implications of user-centric operation are and how the respective functional requirements affect the complexity of $4 \mathrm{G}$ mobile systems is a necessary first step in the pursuit of the user-centric $4 \mathrm{G}$ vision. We have formulated a utility-based model for the "Always Best Connected" vision of 4G mobile communications. We have shown, under different assumptions about the cost of network access, that "Always Best Connected" maps to a class of combinatorial optimization problems that are $N P$-hard. We see future extensions of our work in the simulation of pragmatic scenarios of access network selection problems in $4 \mathrm{G}$ mobile environments, with a focus on the effect of pricing models on the derivation of an optimum solution.

\section{REFERENCES}

[1] International Telecommunicaiton Union (ITU), http://www.itu.ch/.

[2] 4G Forum 2003, 27-28 May 2003, King's College, London, http://www.ctr.kcl.ac.uk/Pages/4GForum/4GForum.htm.

[3] J. Pereira, "Fourth generation - Beyond the hype, a new paradigm", IEE 3G Mobile Communication Technologies, March 28, 2001, London, United Kingdom.

[4] A. K. Dey, "Providing architectural support for building context-aware applications", Ph.D. dissertation, College of Computing, Georgia Institute of Technology, December 2000.

[5] 3GPP Specifications, http://www.3gpp.org/.

[6] V. Gazis, N. Houssos, A. Alonistioti, L. Merakos, "Evolving Perspectives on $4^{\text {th }}$ Generation Mobile Communication Systems", IEEE $13^{\text {th }}$ International Symposium on Personal, Indoor and Mobile Radio Communications (PIMRC 2002), September 2002, Lisbon, Portugal.

[7] C. Xenakis, V. Gazis, L. Merakos, "Secure VPN Deployment in GPRS Mobile Networks", European Wireless 2002, February 25-28, 2002, Florence, Italy.

[8] A, Mas-Collel, M. D. Whinston and J. R. Green, "Microeconomic Theory", Oxford University Press, ISBN 0-19-510268-1.

[9] H. J. Wang, R. H. Katz, J. Giese, "Policy-enabled handoffs across heterogeneous wireless networks", 2nd IEEE Workshops on Mobile Computing and Applications (WMCSA '99), New Orleans, February 1999.

[10] J. Irvine, "Adam Smith goes mobile: Managing services beyond 3G with the digital marketplace", European Wireless 2002, February 25-28, 2002, Florence, Italy.

[11] M. O'Droma et al., "Always Best Connected Enabled 4G Wireless World", IST Mobile Communications Summit 2003.

[12] L.Kleinrock, "On some principles of nomadic computing and multiaccess communications", IEEE Communications Magazine, Vol.38, No 7, pp. 46-50, July 2000.

[13] M. Satyanarayanan, "Mobile Information Access", IEEE Personal Communications Magazine, No. 1, February 1996, pp. 26-33.

[14] S. Khan, "Quality adaptation in a multisession multimedia system: Model, algorithms and architecture", Ph. D. Dissertation, May 1998.

[15] C. Lee, J. Lehoczky, R. Rajkumar, D. Siewiorek, "On QoS optimization with discrete QoS options", IEEE, Real Time Technology and Applications Symposium, 1999.

[16] D. Pisinger, "Algorithms for Knapsack problems", Ph.D. dissertation, Dept. of Computer Science, University of Copenhagen, Denmark. 